\title{
A cutaneous lesion
}

\author{
Pouya Entezari $^{1} \cdot$ Ameneh Alaini $^{2} \cdot$ Hadi Mirfazaelian $^{1} \cdot$ Yahya Daneshbod $^{3}$
}

Received: 16 March 2015/Accepted: 17 March 2015/Published online: 8 April 2015

(C) SIMI 2015

A 34-year-old man presented to dermatology clinic with a lesion on his left palm. The lesion was first noticed 2 weeks before presentation as a nodule with gradual enlargement, accompanied by pain. There was no pruritus or bleeding. He was a shepherd with no prior past medical history. On physical examination, the patient had a $1 \times 1.5 \mathrm{~cm}$ punched out lesion with peripheral purple discoloration on the medial side of the hypothenar region accompanied by erythema and swelling of surroundings (Fig. 1). Except for a mild fever, there were no other abnormal findings including lymphadenopathy. Routine blood test for tularemia and culture of specimen obtained by skin biopsy ruled out Bacillus anthracis. The histology was consistent with the diagnosis of "orf" that was confirmed later by polymerase chain reaction (PCR).

Orf (also known as ecthyma contagiosum) is a worldwide, parapoxvirus zoonotic infection [1]. Contacts with infected animals or contaminated fomites such as barn doors, fences, or shears can spread the virus to humans [2, 3]. After an incubation period of less than 4 weeks, symptoms appear mostly as skin lesions usually in the hands. It can be itchy or painful, and is sometimes accompanied by lymphadenopathy and systematic manifestations [4]. Differential diagnoses are cutaneous anthrax

Hadi Mirfazaelian

h-mirfazaelian@razi.tums.ac.ir

1 Pre-Hospital Reseach Center, Department of Emergency Medicine, Tehran University of Medical Sciences, Tehran, Iran

2 Department of Dermatology, Arak University of Medical Sciences, Arak, Iran

3 Department of pathology, Dr Daneshbod Laboratory, Shiraz, Iran

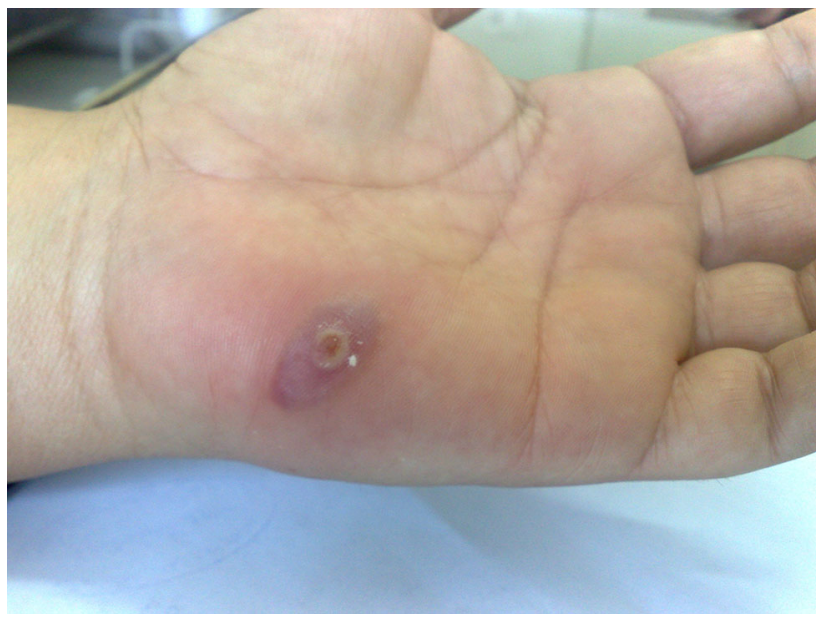

Fig. 1 A punched out lesion with peripheral purple discoloration on the hypothenar region accompanied by erythema and swelling of surroundings

and tularemia [5]. Orf can be differentiated by skin biopsy and PCR (a non-invasive and pathognomonic method in orf diagnosis). In most cases, the lesions are spontaneously resolved without scarring over 6-12 weeks [2]. Severe disease with atypical lesions and protracted course of illness can be seen in immunocompromised individuals [5]. The latter patients need to be treated with antiviral agents [2].

No antiviral agent or antibiotic was prescribed for the patient, and the lesion healed spontaneously without scarring within 5 weeks.

\section{Conflict of interest None.}

Statement of human and animal rights All procedures performed in studies involving participants were in accordance with the ethical standards of the instructional and/or national research committee and with the 1964 Helsinki declaration and its later amendments or 
comparable ethical standards. This article does not contain any studies with animals performed by any of the author.

Informed consent The patient's consent has been obtained.

\section{References}

1. Lederman ER, Green GM, DeGroot HE et al (2007) Progressive ORF virus infection in a patient with lymphoma: successful treatment using imiquimod. Clin Infect Dis 44(11):e100-e103
2. Kitchen M, Muller H, Zobl A et al (2014) ORF virus infection in a hunter in Western Austria, presumably transmitted by game. Acta Derm Venereol 94(2):212-214

3. Nougairede A, Fossati C, Salez N et al (2013) Sheep-to-human transmission of Orf virus during Eid al-Adha religious practices, France. Emerg Infect Dis 19(1):102-105

4. Bassioukas K, Orfanidou A, Stergiopoulou CH et al (1993) Orf. Clinical and epidemiological study. Australas $\mathrm{J}$ Dermatol 34(3):119-123

5. Ara M, Zaballos P, Sanchez M et al (2008) Giant and recurrent orf virus infection in a renal transplant recipient treated with imiquimod. J Am Acad Dermatol 58(2 Suppl):S39-S40 